Check for updates

Cite this: J. Mater. Chem. C, 2021, 9, 2944

Received 30th December 2020 Accepted 27th January 2021

DOI: $10.1039 / \mathrm{d0tc06097f}$

rsc.li/materials-c

\section{Electron spin as fingerprint for charge generation and transport in doped organic semiconductors $\dagger$}

\author{
Alberto Privitera, (D) *a Ross Warren, (D) a Giacomo Londi, (D) ${ }^{\mathrm{b}}$ Pascal Kaienburg, ${ }^{a}$ \\ Junjie Liu, (D) andreas Sperlich, (D) ${ }^{c}$ Andreas E. Lauritzen, (D) ${ }^{a}$ Oliver Thimm, ${ }^{d}$ \\ Arzhang Ardavan, (D) ${ }^{a}$ David Beljonne (D) $^{b}$ and Moritz Riede (D) *a
}

\begin{abstract}
We use the electron spin as a probe to gain insight into the mechanism of molecular doping in a $\mathrm{p}$-doped zinc phthalocyanine host across a broad range of temperatures $(80-280 \mathrm{~K})$ and doping concentrations $(0-5 \mathrm{wt} \%$ of $\mathrm{F} 6-\mathrm{TCNNQ})$. Electron paramagnetic resonance (EPR) spectroscopy discloses the presence of two main paramagnetic species distinguished by two different $g$-tensors, which are assigned based on density functional theory calculations to the formation of a positive polaron on the host and a radical anion on the dopant. Close inspection of the EPR spectra shows that radical anions on the dopants couple in an antiferromagnetic manner at device-relevant doping concentrations, thereby suggesting the presence of dopant clustering, and that positive polarons on the molecular host move by polaron hopping with an activation energy of $5 \mathrm{meV}$. This activation energy is substantially smaller than that inferred from electrical conductivity measurements ( $233 \mathrm{meV}$ ), as the latter also includes a (major) contribution from charge-transfer state dissociation. It emerges from this study that probing the electron spin can provide rich information on the nature and dynamics of charge carriers generated upon doping molecular semiconductors, which could serve as a basis for the design of the next generation of dopant and host materials.
\end{abstract}

\section{Introduction}

The precise control of the charge carrier density and conductivity of organic semiconductors through molecular doping has been critical for the successful commercialisation of organic light emitting diodes (OLEDs) and has been used to improve the performance of organic solar cells (OSCs) and organic fieldeffect transistors (OFETs). ${ }^{1-3}$ Despite the commercial success of molecular doping in the multi-billion dollar OLED industry, some fundamental aspects concerning charge generation and transport mechanisms are not yet sufficiently understood. ${ }^{3,4}$ In the last few years, molecular doping has attained renewed interest sparked by the recent advancements that clarified the elementary steps occurring after the introduction of dopant molecules inside organic layers, and the rationalization of the

\footnotetext{
${ }^{a}$ Clarendon Laboratory, Department of Physics, University of Oxford,

Oxford OX1 3PU, England, UK. E-mail: alberto.privitera@physics.ox.ac.uk, moritz.riede@physics.ox.ac.uk

${ }^{b}$ Laboratory for Chemistry of Novel Materials, University of Mons, B-7000 Mons, Belgium

${ }^{c}$ Experimental Physics VI, Julius Maximilian University of Würzburg, 97074 Würzburg, Germany

${ }^{d}$ IEK5-Photovoltaics, Forschungszentrum Jülich, 52425 Jülich, Germany

$\dagger$ Electronic supplementary information (ESI) available. See DOI: 10.1039/ dotc06097f
}

thermally-activated charge carrier transport. ${ }^{5-11}$ This has allowed for a better control of the doping process and has demonstrated that a complete understanding of molecular doping is essential to develop novel dopant molecules with appropriately-tailored energetic levels and push forward the performance of organic devices in which doped layers play crucial roles. ${ }^{5,12-16}$

The most recent doping model consists of three steps, as schematically shown in Fig. 1 for the case of efficient p-type doping. ${ }^{6,17}$ The first step involves either host-dopant frontier molecular orbitals hybridization or ground-state integer-charge transfer (ICT) from donor to acceptor molecules. Ground-state ICT appears more effective to achieve high doping efficiency and constitutes the main subject of our research interest. Ground-state ICT generates a coulombically bound electronhole pair, known as charge transfer (CT) state (Fig. 1a). ${ }^{9,10}$ The second step consists in the subsequent dissociation of the formed CT state and the generation of separated charges carriers (Fig. 1b) ${ }^{6,18}$ To prevent later confusion, in this work, the positively charged species, both bound $\left(\mathrm{N}_{\mathrm{CT}}{ }^{+}\right)$and separated $(p)$ ones, are referred as positive polarons because of the delocalized nature of the interaction between the hole and the host lattice. Conversely, the negatively charged species, both bound and separated ones $\left(N_{\mathrm{A}}^{-}\right)$, are referred as radical anions since, as discussed in following, the electron is mostly 
a)

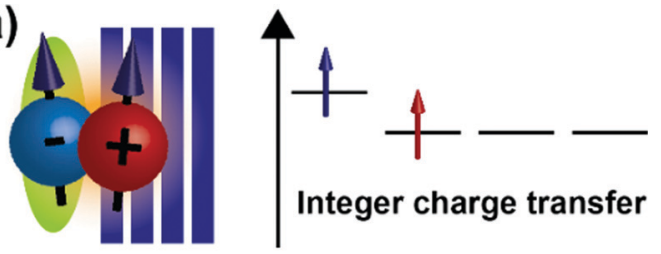

b)
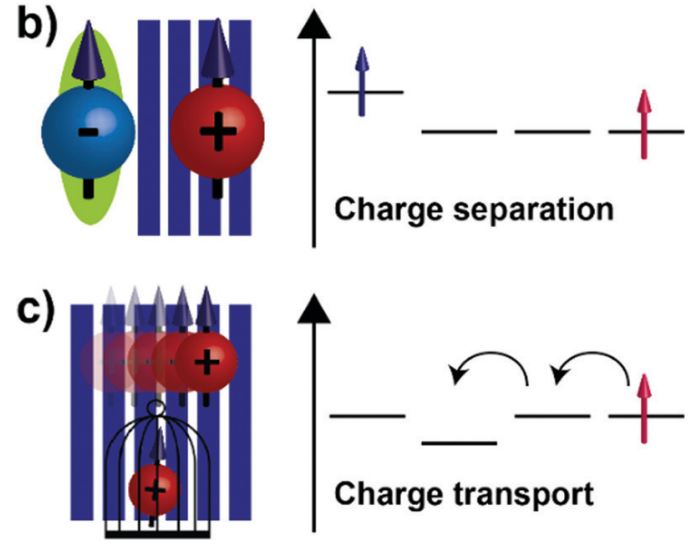

Fig. 1 Schematic representation of efficient molecular doping for host material ZnPc (blue rectangles) with p-type dopant F6-TCNNQ (green oval). (a) Firstly, a charge transfer (CT) state is generated by integer electron transfer from the highest occupied molecular orbital (HOMO) of ZnPc to the lowest unoccupied molecular orbital (LUMO) of F6-TCNNQ. The CT state consists of a hole localized on the $\mathrm{ZnPc}$ molecule and an electron localized on the dopant molecule, bound by an attractive Coulomb interaction. (b) Secondly, the bound charge carriers (F6-TCNNQ anion and $\mathrm{ZnPc}$ positive polaron) overcome the binding energy and dissociate, thereby forming a charge separated (CS) state. (c) Thirdly, the separated charge carriers can either move within the host material by a hopping process or remain static in trap states.

localized on the dopant molecule. In the third step, after charge dissociation, the separated positive polarons can either move within the host material, likely by a hopping process, reflecting the disordered nature of organic semiconductors, or remain static in trap states (Fig. 1c). ${ }^{17}$ The balance between mobile and trapped species, as well as the concentration and temperature dependence of doping efficiency, has a statistical nature and has been described by Tietze et al. . $^{6,17}$

All three steps in Fig. 1 are characterized by the presence of unpaired electron spins $(S=1 / 2)$, as a result of the ground-state ICT process that generates an unpaired electron spin both on the donor and acceptor molecules. ${ }^{13,19-24}$ Taking advantage of the electron spin to investigate molecular doping represents a new perspective from which to study charge generation and transport in doped layers, since the electron spin is sensitive to the magnetic interactions acting at the microscopic scale allowing for new insights into molecular doping. In this context, electron paramagnetic resonance (EPR) spectroscopy provides a significant advantage to study molecular doping since it allows the observation of only the spin-bearing species and is unaffected by the diamagnetic species (e.g. non-ionized host and dopant molecules), whose contribution often overlaps the signals of the polarons in optical absorption techniques. ${ }^{24-31}$ Although EPR spectroscopy has been used to study polarons and their microscopic dynamics in different $\pi$-conjugated organic materials, a comprehensive picture that takes into account the first steps of charge generation in doped organic semiconductors and the effect of doping concentration and temperature on the number, the chemical environment and the microscopic dynamics of polarons is so-far missing. ${ }^{13,19-30}$

Here, we investigate p-type doping by establishing new connections between spin physics, through electron paramagnetic resonance (EPR) spectroscopy and theoretical density functional theory (DFT) calculations, and more conventional characterisation methods, such as photothermal deflection spectroscopy (PDS), electrical measurements, morphological analysis and transport models. We carry out our analysis on a model system of zinc-phthalocyanine ( $\mathrm{ZnPc})$, as the host material, co-evaporated with the p-type dopant F6-TCNNQ at different doping concentrations $(0-5 \mathrm{wt} \%$, molar ratio $(\mathrm{MR})=$ $0-0.085)$ and measured at different temperatures $(80-280 \mathrm{~K})$. The EPR and DFT analysis shows the presence of two different paramagnetic species, whose $g$-values are attributable to the F6-TCNNQ radical anion $\left(g_{\text {iso }}=2.0034 \pm 0.0005\right)$ and the $\mathrm{ZnPc}$ positive polaron $\left(g_{\text {iso }}=2.0023 \pm 0.0005\right)$. The EPR quantitative analysis and DFT simulations shed light on the charge generation mechanism in doped semiconducting organic films and suggest an antiferromagnetic coupling mechanism occurring among F6-TCNNQ radical anions at high doping concentrations. The investigation of polaron motional dynamics from the EPR line shapes clarifies the microscopic transport mechanism of polarons based on a thermallyactivated $\left(E_{\mathrm{A}}=5 \pm 1 \mathrm{meV}\right)$ hopping mechanism. All EPR results are compared with electrical conductivity measurements that instead show an activation energy of $233 \pm 1 \mathrm{meV}$. We associate this larger activation energy to a dominant contribution arising from CT state dissociation, while both bound and free polarons contribute to EPR.

\section{Experimental section}

\subsection{Sample preparation}

All the samples were prepared using vacuum deposition. ZnPc (Zinc phthalocyanine) was purchased from Luminescence Technology Corp., Taiwan, and F6-TCNNQ (1,3,4,5,7,8hexafluoro-tetracyanonaphthoquinodimethane) from Novaled $\mathrm{GmbH}$, Germany. All organic molecules were purchased in sublimed grade and used as received.

Films for cw EPR were processed on glass substrates (Brand microscope cover glasses, $18 \times 18 \times 0.15 \mathrm{~mm}^{3}$ ). Substrates were cut in stripes (width $=3 \mathrm{~mm}$ ) using a diamond tipped glass cutter and cleaned by sequential sonication in detergent (Hellmanex $\mathrm{GmbH}$ ), de-ionised water (DI), acetone and iso-propanol (IPA) for 10 minutes each and finally UV-ozone treated for 10 minutes. Thin films were evaporated onto the substrate in a custom vacuum deposition tool (Creaphys GmbH, Germany, base pressure $=10^{-6}-10^{-7} \mathrm{mbar}$ ) and transferred to a nitrogenfilled glovebox without air exposure. The deposition rate $\left(\approx 0.4 \AA^{-1} \mathrm{~s}^{-1}\right)$ was controlled by quartz crystal microbalances 
calibrated by X-ray reflectivity measurements. For doped films, the relative deposition rate of the materials was varied to obtain the desired ZnPc:F6-TCNNQ ratio. The samples were then inserted within quartz EPR tubes (inner diameter $=3 \mathrm{~mm}$ ) and sealed with a two-part epoxy adhesive (UHU Plus Endfest).

\subsection{EPR measurements}

The cw EPR spectra were recorded on a Bruker Elexsys E680 $\mathrm{X}$-band spectrometer equipped with a nitrogen gas-flow cryostat for sample temperature control. The sample temperature was maintained with an Oxford Instruments CF9350 cryostat and controlled with an Oxford Instruments ITC503. Unless specified in the text, the following experimental parameters were used in all cw EPR measurements: modulation amplitude $=1 \mathrm{G}$, microwave power $=0.2 \mathrm{~mW}$ (30 dB attenuation).

EPR spectral simulations were carried out using the routines of Easyspin, a Matlab ${ }^{\mathrm{TM}}$ toolbox. $^{32}$

All the films studied via EPR spectroscopy were fabricated with the same thickness (100 $\mathrm{nm}$ ) to allow a better comparison of the acquired EPR spectra and a correct estimate of the concentration of the paramagnetic species.

\subsection{DFT calculations}

The $g$-tensor values were calculated for a negative charged F6-TCNNQ molecule sandwiched between two neutral ZnPc molecules along the ZnPc stacking direction, at a distance of $3.77 \AA$ from the anion. The calculations were carried out using the PBE0 functional and the Def2-TZVP basis set for all the atomic elements. ${ }^{33}$ We also took advantage of the RIJCOSX approximation along with the Def2/J and Def2-TZVP/C auxiliary basis set, as implemented in the ORCA 4.2.1 software. ${ }^{34}$ The same level of theory was used for the energy difference calculations between a triplet and a singlet state in two F6-TCNNQ anions. In order to describe this system, the Heisenberg-Dirac-vanVleck (HDvV) Hamiltonian for two interacting magnetic centres with localized spin $S_{1}$ and $S_{2}$ reads:

$$
H_{\mathrm{HDvV}}=-2 J_{12} S_{1} \cdot S_{2}
$$

If the parameter $J_{12}>0$, the two centres are ferromagnetically coupled and the highest spin-state is the lowest in energy, while if $J_{12}<0$, the coupling is antiferromagnetic and the lowest spin-state is the most stable in energy. In our case, the energies of the states with $S_{\max }=1$ (a triplet state) and $S_{\min }=0$ (a singlet state) have to be computed. If, on one hand, the energy of the state with $S_{\max }$ is well described by one single Slater determinant using a spin unrestricted formalism, on the other hand, the determination of the energy of the state with $S_{\text {min }}$ cannot be exactly computed with DFT, due to the singledeterminant nature of the Kohn-Sham implementation, but only using multiconfiguration (i.e. MR-SCF) approaches. ${ }^{35}$ Nevertheless, at the DFT level, a possible work-around to such a problem is the so-called "Broken Symmetry (BS) approach", where the energy of the lowest spin-state (a pure spin-state) is approximately computed as a projection from a state of mixed spin and spatial symmetry (the BS state) obtained by an independent SCF calculation. Then, the following expression can be used:

$$
\Delta E_{\mathrm{T}-\mathrm{S}}=E(\mathrm{HS})-E(\mathrm{BS}) \propto-J_{12}
$$

\subsection{Photothermal deflection spectroscopy}

Films of $40 \mathrm{~nm}$ thickness were deposited on quartz substrates and mounted in a cuvette containing the liquid FC75 without any exposure to ambient atmosphere. A monochromatic beam of light directed perpendicularly at the sample was obtained from a xenon lamp followed by a monochromator. The absorption of photons in the probed material causes heating of the sample which subsequently heats the surrounding liquid. FC75's refractive index is highly temperature sensitive and the change in refractive index caused by heating was detected via the deflection of a laser beam that runs parallel to the sample surface. The measurement signal at a quadrant photodiode is proportional to the absorptance of the thin film from which the absorption coefficient was calculated using the sample thickness. ${ }^{36,37}$ Improved signal-to-noise ratio was achieved by chopping the monochromatic light and lock-in detection.

\subsection{Electrical measurements}

Electrical conductivity measurements were performed on Fraunhofer IPMS gen 4 OFET substrates with $230 \mathrm{~nm} \mathrm{\textrm {SiO } _ { 2 }}$ and gold interdigitated electrodes. Measurements were made over channel lengths $2.5,5,10$ and $20 \mu \mathrm{m}$. The transmission line method was used to check that the contact resistance was significantly lower than the sample resistance. Room temperature measurements were made under a nitrogen atmosphere, with the samples having had no exposure to air. A nitrogen cryostat was used to cool the samples for measurements at low temperature.

\subsection{Grazing-incidence wide-angle $X$-ray scattering}

Grazing-incidence wide-angle X-ray scattering (GIWAXS) studies are carried out during experiment SI20426-1 at the Surface and Interface Diffraction beamline (I07) at the Diamond Light Source (DLS) using a beam energy of $20 \mathrm{keV}(0.62 \AA)$ and a Pilatus2M area detector. The samples are probed while inside a vacuum deposition chamber at a pressure of around $10^{-3} \mathrm{mbar}$ with the MINERVA setup. ${ }^{38}$ The sample-to-detector distance was $42.1 \mathrm{~cm}$ as determined via AgBeh calibration. Images are converted to 2D reciprocal space using the DAWN software package with an applied polarisation and solid angle correction. The GIWAXS figures were previously published in ref. 39 and more details can be found there.

\section{Results and discussion}

As a baseline, we carried out reference EPR measurements on a pure $\mathrm{ZnPc}$ film in the dark at $280 \mathrm{~K}$ to confirm the absence of any paramagnetic species or impurities in the host material. The corresponding EPR spectrum shown in Fig. SI1 (ESI $\dagger$ ) does not present any appreciable signal, confirming that there are no detectable spins which could arise from partial intrinsic 
oxidation of ZnPc pristine material or unintentional doping due to the stock conditions. ${ }^{22,26}$

\subsection{Two spin-bearing species generated by doping}

The EPR spectrum of ZnPc doped with $1 \mathrm{wt} \%(\mathrm{MR}=0.017)$ of the acceptor F6-TCNNQ, reported in Fig. 2a, shows two different peaks with a narrow line shape and a field position, which are typical of organic radicals. ${ }^{31}$ The presence of two EPR peaks was attributed to two different paramagnetic species in the film, as confirmed by saturation measurements (Fig. SI2, ESI $\dagger$ ). From the best-fit spectral simulations (Fig. 2a), the $g$-values of the two species were obtained $\left(g_{1}=2.0034 \pm 0.0005\right.$ and $g_{2}=2.0023 \pm$ 0.0005). Comparing the $g$-values obtained from simulation with literature values, we attribute species 1 as the radical anion localized on F6-TCNNQ and species 2 as the positive polaron on ZnPc. ${ }^{40-42}$ Given that the EPR signals of the two paramagnetic species overlap and the literature value for the radical anion of F6-TCNNQ is missing, the obtained $g$-value for F6-TCNNQ represents only a rough estimate and does not provide any information on its anisotropic nature. We therefore carried out DFT calculations to get a deeper insight into the F6-TCNNQ $g$-tensor (Table SI1 and Section SI-3 for further details, ESI $\dagger$ ). The calculated isotropic $g$-value $\left(g_{\text {iso }}=2.0034\right)$ matches the EPR result, supporting our analysis. In addition, DFT calculations clearly show the anisotropic character of the F6-TCNNQ $g$-tensor $\left(g_{x}=2.0026, g_{y}=2.0034, g_{z}=2.0041\right)$, which cannot be inferred by EPR measurements in X-band. The anisotropy of the $g$-value could be disclosed by using higher-field EPR spectroscopy (e.g. W-band), which is outside the scope of our work and not relevant for our conclusions. The calculated DFT $g$-tensor values are used for all the best-fit spectral simulations carried out in the following (further details in SI-4, ESI $\dagger$ ). From the spectral simulation, we obtained the relative EPR intensities (double integral of the EPR line) for each species (Table SI2, ESI $\dagger$ ). The obtained values suggest an equal number of $\mathrm{ZnPc}$ positive polarons and F6-TCNNQ anions in the sample at $1 \mathrm{wt} \%$ doping concentration. These initial EPR results are consistent with the first step of the doping mechanism outlined above. Namely, when ZnPc is doped with the electron acceptor F6-TCNNQ, a ground-state ICT occurs that results in an anion localized on the F6-TCNNQ and a positive polaron on the ZnPc. These two species are both paramagnetic $(S=1 / 2)$ and therefore detectable through EPR. Notably, the F6-TCNNQ anion and the ZnPc positive polaron can be either bound (CT state) or separated (CS state), as shown in Fig. 1a and b. The EPR signal is the sum of both contributions.

The line width of the EPR signal can provide a qualitative estimate of the average microscopic mobility of the studied species, which we refer to as $\mu_{\mathrm{EPR}}$ in the following. ${ }^{43,44}$ Static
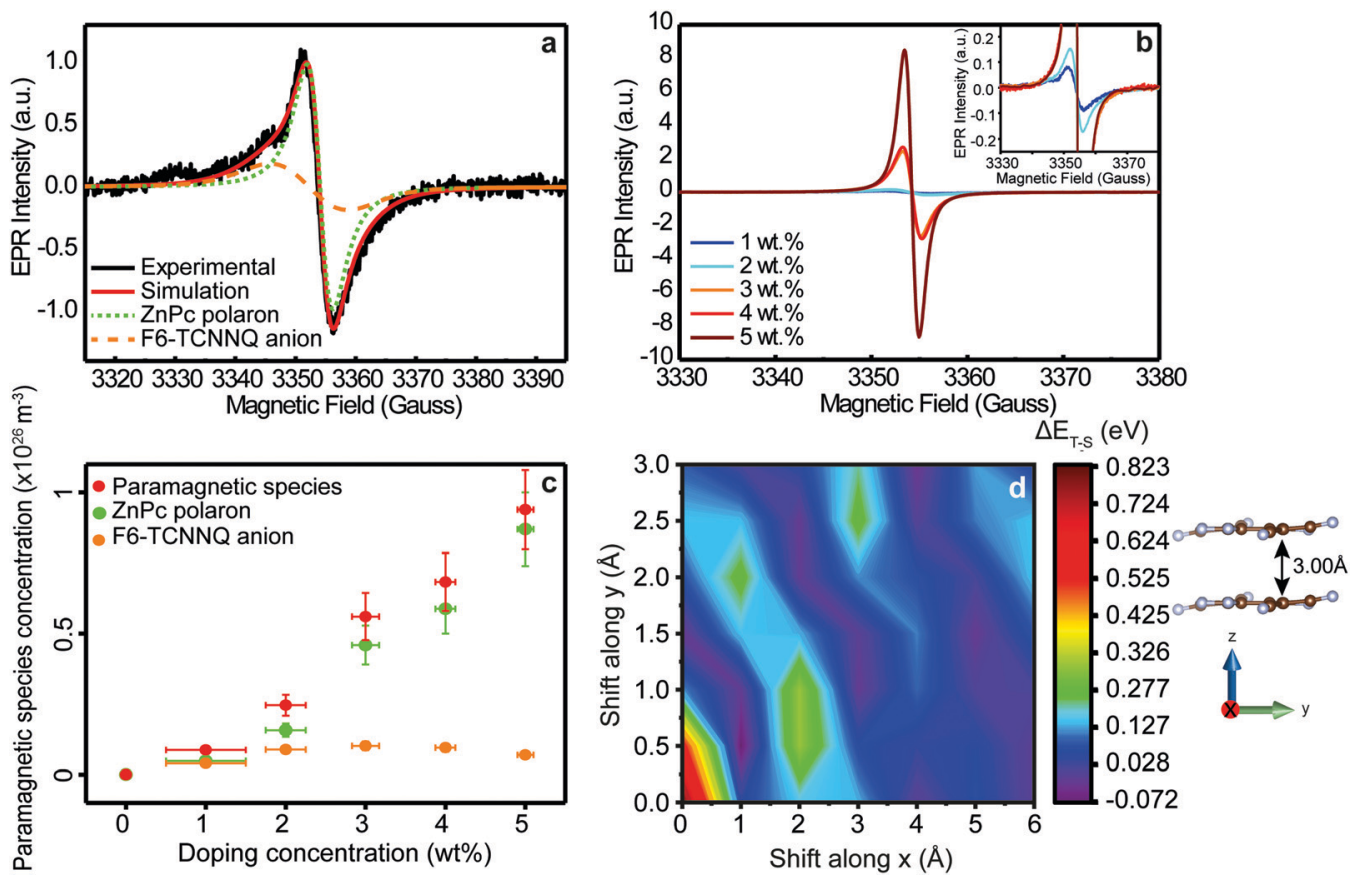

Fig. 2 Spin generation and antiferromagnetic coupling mechanism. (a) Black line: $c w$ EPR spectrum of ZnPc doped with 1 wt\% (MR = 0.017 ) of F6-TCNNQ recorded at $280 \mathrm{~K}$. Red line: Spectral simulation of the EPR spectrum obtained as the sum of two contributions: F6-TCNNQ anion (dashed orange line) and ZnPc positive polaron (dotted green line). (b) cw EPR spectra of ZnPc doped with F6-TCNNQ at different concentrations, from 1 wt\% to $5 \mathrm{wt} \%$ (MR $=0.017-0.085)$. The spectra were recorded at $280 \mathrm{~K}$. (c) Paramagnetic species concentration (red dots), positive ZnPc polaron concentration (green dots, $\mathrm{N}_{\mathrm{CT}^{+}}{ }^{+} p$ ) and $\mathrm{F} 6-\mathrm{TCNNQ}$ anion concentration (orange dots, $\mathrm{N}_{\mathrm{A}}^{-}$) as a function of doping concentration, from $1 \mathrm{wt} \%$ to $5 \mathrm{wt} \%$ (MR $=0.017-$ $0.085)$, calculated by EPR quantitative analysis of spectra in Fig. 2b. The paramagnetic species concentration is the sum of ZnPc polarons and F6-TCNNQ anion concentrations. (d) $2 \mathrm{D}$ contour plot of the energy difference between the triplet and the singlet states ( $\Delta E_{\mathrm{T}-\mathrm{s}}$ ) of two coupled electrons localized on nearby F6-TCNNQ molecules, as a function of the $x$ and $y$ relative displacement of one molecule with respect to the other (see Section SI-9 for details, ESI†). The two molecules are distant by $3.00 \AA$ along the $z$ axis. A total of $7 \times 7$ configurations were probed with Broken-Symmetry (BS) DFT calculations. 
species usually possess a Gaussian-broadened and anisotropic line shape that reflects local inhomogeneity in chemical and magnetic environment. ${ }^{43}$ For species with higher mobilities, the line shape becomes Lorentzian and narrower (motional narrowing regime) as the signal tends towards the mean of the distribution. ${ }^{45}$ In our samples, the line width of the positive polaron on $\mathrm{ZnPc}$ is on average much narrower than the line width of the F6-TCNNQ anion (Table SI2, ESI $\dagger$ ). This suggests that positive polarons, either bound or separated, have on average higher mobility than the counterpart radical anion. This result is further corroborated by considering the Lorentzian nature of the ZnPc positive polaron EPR line, which confirms the motional narrowing regime. Lower mobility of the F6-TCNNQ anion is rationalized considering the large hopping distances between F6-TCNNQ dopant molecules, given their lower number compared to $\mathrm{ZnPc}$ host molecules.

\subsection{Antiferromagnetic coupling mechanism}

After having identified the nature of the paramagnetic species generated by the electron transfer process, we focused our attention on the effect of increasing doping concentrations. The EPR spectra of ZnPc doped with F6-TCNNQ at different concentrations (from $1 \mathrm{wt} \%$ to $5 \mathrm{wt} \%, \mathrm{MR}=0.017-0.085$ ), recorded at $280 \mathrm{~K}$, are reported in Fig. $2 \mathrm{~b}$. The spectra at all concentrations show the presence of the F6-TCNNQ radical anion and the ZnPc positive polaron, as further corroborated by the best-fit spectral simulations (Fig. SI4, ESI $\dagger$ ) and the discussion in the ESI $\dagger$ (see SI-5). Even though this result seems in line with the ground-state ICT between host and dopant, the relative ratio of the EPR intensities of the two paramagnetic species deviates substantially from 1:1 at higher doping concentrations. To get a better understanding for the unexpected behaviour of the anion, we carried out EPR quantitative analysis. From the EPR intensity (double integral of EPR line), it is possible to calculate the concentration of $\mathrm{ZnPc}$ positive polarons (both bound and separated) in the doped film, and of F6TCNNQ anions, as reported in Fig. 2c. The details of the quantitative analysis are described in the ESI $\dagger$ (see SI-6). The quantitative EPR analysis underlines that the concentration of ZnPc positive polarons $\left(N_{\mathrm{CT}}{ }^{+}+p\right)$ increases monotonically with doping level as expected, while the concentration of F6-TCNNQ anions $\left(N_{\mathrm{A}}^{-}\right)$shows a non-monotonic trend with a maximum at $3 \mathrm{wt} \%(\mathrm{MR}=0.052)$ dopant concentration. To confirm this result, we performed photothermal deflection spectroscopy (PDS) at room temperature for the same doping concentrations studied with EPR (Fig. SI7, ESI $\dagger$ ). PDS allows for the detection of sub-band gap states whose absorption coefficients are usually very low. ${ }^{46}$ Analogously to previous results,${ }^{39}$ from Fig. SI6 (ESI $\dagger$ ) two main peaks are observed in the absorption region below the $\pi-\pi$ transitions of phthalocyanine's Q-band: one at $1.06 \mathrm{eV}(1170 \mathrm{~nm})$ and one at $1.24 \mathrm{eV}(1000 \mathrm{~nm})$. By plotting the absorption coefficients of the peak at $1.06 \mathrm{eV}$ (or alternatively $1.24 \mathrm{eV}$ ), which is attributed to the F6-TCNNQ anion, ${ }^{6}$ a relative quantification of the number of F6-TCNNQ anions in the film can be obtained (Fig. SI7-S9, ESI $\dagger$ ). The absorption coefficients show the same non-monotonic trend as a function of the dopant concentration as observed via EPR, supporting the EPR quantitative analysis. Further discussion of PDS analysis is reported in Section SI-7 of ESI. $\dagger$

We rationalize the trend of the F6-TCNNQ anion signal as follows: after the ground-state electron transfer process, the number of ZnPc positive polarons and F6-TCNNQ anions is equal because of the conservation of charge. This means that for each ZnPc positive polaron generated, a negative F6-TCNNQ anion is created as well. Each species possesses an unpaired spin. At low doping concentrations, the distances between the spin-bearing species are usually large and no magnetic interaction occurs among them. In this case, both species contribute equally to the EPR signal. Conversely, if the distance between two spin-bearing species decreases and becomes small enough, a coupling between the two magnetic centres can occur. In the latter scenario, two spins can couple either in a ferromagnetic (where the triplet state has a lower energy than the singlet state, $\Delta E_{\mathrm{T}-\mathrm{S}}=E_{\mathrm{T}}-E_{\mathrm{S}}<0$ ) or in an antiferromagnetic (where the singlet state has a lower energy, i.e. $\Delta E_{\mathrm{T}-\mathrm{S}}>0$ ) fashion. In the antiferromagnetic coupling case, the total spin $S$ is equal to zero and the coupled spins do not contribute anymore to the overall EPR signal, as this is only sensitive to unpaired spins. Such a magnetic coupling is expected to appear at higher doping concentrations where dopant molecules are more likely to be close to each other, due for example to clustering. ${ }^{36,47}$ We thus hypothesize that, when two F6TCNNQ anions are localized on nearby molecules, the two unpaired spins magnetically interact in an antiferromagnetic way thus turning these paired spins EPR silent. To demonstrate our hypothesis, we carried out DFT calculations on two negatively charged F6-TCNNQ molecules, applying the Broken Symmetry (BS) formalism (see Methods and Section SI-9 for details, ESI $\dagger$ ). ${ }^{48}$ In absence of direct structural or morphological information about dopant clustering, we first assumed a cofacial arrangement of the F6-TCNNQ molecules with an intermolecular separation of $3.00 \AA$ along the $z$ axis (see Fig. 2d), in accordance with structural reviews and computational studies by Miller ${ }^{49}$ on related TCNQ and TCNE radical anion salts and further structural studies on TCNQ derivatives salts by Sutton. ${ }^{41}$ Then, we assessed the energy difference between the highest spin state, i.e. the triplet state, and the BS state, i.e. a non-total symmetric singlet state, as a function of sliding one F6-TCNNQ molecule with respect to the other in the $x$ and $y$ directions. The theoretical results support the view that an antiferromagnetic arrangement of the coupled spins $\left(\Delta E_{\mathrm{T}-\mathrm{S}}>0\right)$ is preferred over a broad range of molecular packing arrangements (with only few structures yielding a very small, negative singlet-triplet gap, shown as purple regions in Fig. 2d). This hypothesis is further corroborated indirectly from EPR and PDS analysis, while other possible scenarios like MW power saturation or di-anion formation are ruled out (see SI-5, ESI $\dagger$ ). At this point, it is worth mentioning that the proposed antiferromagnetic mechanism appears to be relevant also at relatively low doping concentrations (MR $=0.034)$, which can occur only if an inhomogeneous dopant distribution is taken into account. In this regard, we performed statistical simulations which suggest that in our doped $\mathrm{ZnPc}$ 

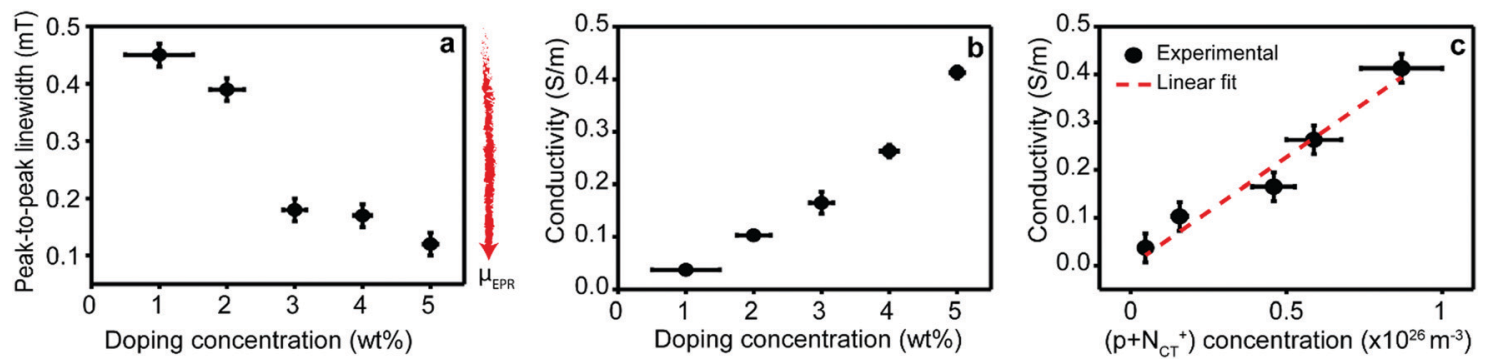

Fig. 3 Microscopic vs. macroscopic transport dynamics. (a) Lorentzian peak-to-peak line widths obtained from spectral fits of the EPR signals of ZnPc polarons reported in Fig. $2 \mathrm{~b}$ and Fig. SI4 (ESI†) as a function of doping concentration. The EPR peak-to-peak line width is inversely proportional to $\mu_{\mathrm{EPR}}$ (red arrow: direction of increasing microscopic mobility). (b) Conductivities of ZnPc layers doped with F6-TCNNQ as a function of doping concentration. (c) Conductivity (dots) of ZnPc layers doped with F6-TCNNQ as a function of polaron concentration $\left(\mathrm{N}_{\mathrm{CT}}{ }^{+}+p\right)$ obtained via EPR quantitative analysis and best linear fit (red line). The values obtained from the linear fit are reported in Section SI-10 of ESI.†

films, dopants tend to cluster as further discussed in Section SI-8 of ESI. $\dagger$ More generally, our results provide a promising route for further investigations into dopant clustering in organic semiconductors, an effect that is often difficult to determine at device relevant doping concentrations. All in all, this antiferromagnetic coupling mechanism, which to our knowledge has never been mentioned in literature, appears intriguing since it occurs also at room temperature and at relatively low, but device-relevant, doping concentrations $(\sim$ few $\%)$ and suggests that, if further investigated, molecular doping can play an important role in the exciting field of organic spintronics.

\subsection{Microscopic vs. macroscopic transport dynamics}

In addition to the EPR intensity, additional information can be obtained from the EPR line widths in Fig. 3a. Here, we focus our attention on the dominant $\mathrm{ZnPc}$ polaron contribution to track the electrical properties of our films as a function of doping level. Specifically, the EPR line width drops with increasing doping concentration, implying an increase in the average microscopic polaron mobility. ${ }^{27,30}$

We performed electrical conductivity measurements of the ZnPc films doped with F6-TCNNQ to verify the effect observed by the EPR measurements. Fig. 3b shows the conductivity plotted against doping concentrations $1-5 \mathrm{wt} \%$ (MR $=0.017-$ 0.085). The increase in conductivity with doping concentration is in excellent agreement with the increase in polaron density observed via EPR. To further elucidate this point, the linear fit of conductivity as a function of polaron density and the best-fit values are reported in Fig. 3c and Section SI-10 (ESI $\dagger$ ), respectively. The linear fit shows a strong positive correlation $\left(R_{\text {adj. }}=\right.$ 0.984 ) thereby confirming the linear dependence between the conductivity and the polaron density. The absence of deviations from the linear relationship highlights that both the ratio between bound and free polarons $\left(N_{\mathrm{CT}}{ }^{+} / p\right)$ and the macroscopic mobility $(\mu)$ of the polarons are constant, as discussed in SI-10 (ESI $\dagger$ ). On the one hand, the constant $N_{\mathrm{CT}}^{+} / p$ ratio is in line with the Tietze model ${ }^{6}$ for the studied doping range and underlines that the charge separation efficiency of the CT state does not significantly change in the studied doping range. On the other hand, the constant electrical mobility $(\mu)$ obtained from conductivity measurements appears at first glance inconsistent with the changes in polaron transport $\left(\mu_{\mathrm{EPR}}\right)$ probed with EPR. The key to understand these different behaviours is that electrical conductivity results from macroscopic charge transport phenomena that covers processes over several length scales, while EPR only probes the microscopic movement of charges originating from local fluctuations in the environment of the charges within the EPR time resolution. In addition, both bound and free polarons contribute to EPR, while electrical conductivity is sourced only by the free holes. This result is further corroborated and clarified through temperaturedependent measurements below.

\subsection{Spin-bearing species as a function of temperature (80-280 K)}

To study the effect of temperature on the equilibrium and the dynamics of the spin-bearing species, similar EPR measurements were performed on the same samples at different temperatures $(80-280 \mathrm{~K})$, as reported in Fig. SI14 (ESI $\dagger$ ). The spectra at lower temperatures appear similar to those recorded at $280 \mathrm{~K}$ and confirm the presence of the same two paramagnetic species, previously discussed, at all temperatures. From the best-fit spectral simulations, the relative weights of the EPR intensities of the $\mathrm{ZnPc}$ positive polaron $\left(c_{\mathrm{A}}=p+\frac{N_{\mathrm{CT}^{+}}}{p+N_{\mathrm{CT}^{+}}+N_{\mathrm{A}^{-}}}\right)$and F6-TCNNQ anion $\left(1-c_{\mathrm{A}}\right)$ were obtained (Fig. SI15, ESI $\dagger$ ). From the analysis, the polaron ratio $\left(c_{\mathrm{A}}\right)$ does not seem to significantly depend on temperature. This result underlines that although the transition from the antiferromagnetic coupling regime to the paramagnetic regime is in principle thermally activated, its activation energy is high compared to the thermal energy at room temperature $\left(k_{\mathrm{BT}} \approx\right.$ $25 \mathrm{meV})$. This conclusion is further corroborated by the $\Delta E_{\mathrm{T}-\mathrm{S}}$ values obtained by DFT calculations (Fig. 2d). Conversely, the ratio is clearly dependent on the doping concentration (Fig. SI15, ESI $\dagger$ ), given that at higher doping concentrations dopant clustering is more likely leading to a reduction of the distance between unpaired spins.

Since the EPR intensity (double integral of the EPR spectra) is proportional to the spin susceptibility of the sample, the investigation of the EPR intensity of ZnPc positive polaron as a function of the temperature provides insight on how the 

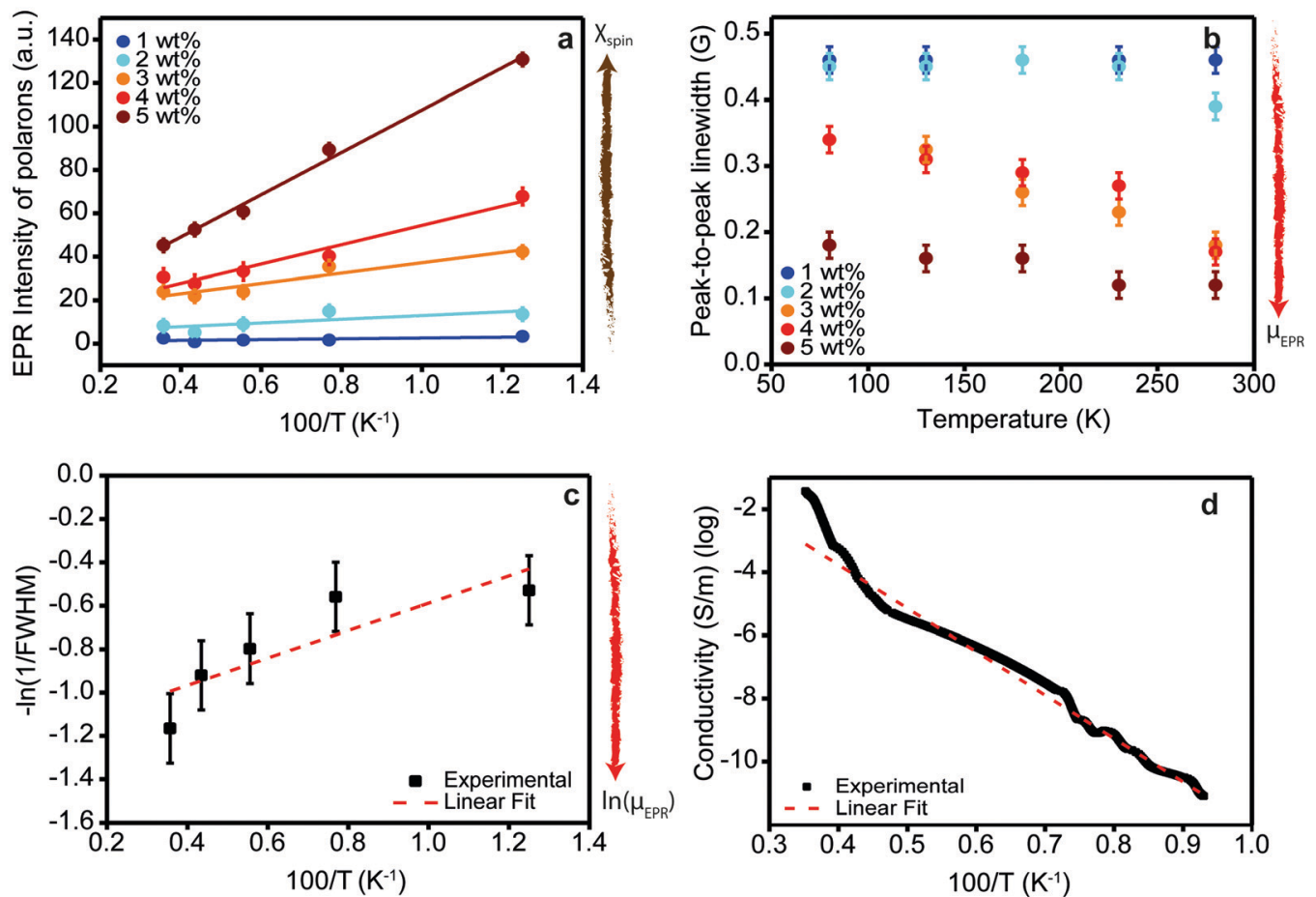

Fig. 4 Spin susceptibility and charge transport as a function of temperature. (a) EPR intensity (dots) of ZnPc films doped with F6-TCNNQ at different concentrations $(1-5 \mathrm{wt} \%, \mathrm{MR}=0.017-0.085)$ as a function of the inverse of the temperature and best linear fit (dotted lines). The EPR intensity is directly proportional to the spin susceptibility (brown arrow: direction of increasing spin susceptibility). The values obtained from the linear fit are tabulated in Table SI3 (ESI $\dagger$ ). The EPR intensities have been calculated by performing the double integral of spectra in Fig. SII4 (ESI†) and taking into account the weighting factor $c_{A}$. (b) Peak-to-peak line widths of the polaron contribution of EPR spectra in Fig. SI14 (ESI $\dagger$ ) as a function of temperature at different doping concentrations. The EPR peak-to-peak line widths, which in the motional narrowing regime are inversely proportional to $\mu_{\mathrm{EPR}}$ (red arrow: direction of increasing microscopic mobility), have been obtained by the best-fit spectral simulations of spectra in Fig. SI14 (ESI†). (c) FWHM (dots) of the polaron contribution of the EPR spectrum of $3 \mathrm{wt} \%(M R=0.052)$ sample as a function of temperature. FWHM is related to the peak-to-peak Lorentzian line width by the following relationship: $P 2 P=\mathrm{FWHM} / \sqrt{3}$. The linear fit (red line) provides the activation energy for the intra-grain polaron hopping $E_{\mathrm{A}}=$ $(5 \pm 1)$ meV. (d) Electrical conductivity (dots) of ZnPc doped with 3 wt\% (MR $=0.052)$ of F6-TCNNQ as a function of the inverse of the temperature. The linear fit (dotted red line) provides the thermal activation energy of the conductivity.

number of polarons and ultimately the electron transfer process are affected by temperature. ${ }^{25}$ In Fig. 4a, we report the EPR intensities of $\mathrm{ZnPc}$ positive polarons at different doping concentrations as a function of the inverse of the temperature. In the absence of couple-exchange spin pairs, the spin susceptibility for an organic semiconductor can be well rationalized by the sum of two contributions: the Curie (proportional to $N / T$, where $N$ is the number of spin-bearing species and $T$ is the temperature) and the Pauli terms (temperature-independent). ${ }^{25,44}$ The Curie term arises from isolated spin-bearing species. In contrast, the Pauli term is typical of conduction electrons or holes and might play an important role at very high doping concentrations where the behaviour of the doped film resembles the behaviour of metal. ${ }^{25}$ We performed a linear fitting of EPR intensities as a function of $1 / T$ in order to differentiate these two contributions $\left(I_{\mathrm{EPR}}=A+B / T\right.$, where $A$ is the Pauli term and $B$ the Curie term). From the analysis, whose values are reported in Table SI3 (ESI $\dagger$ ), we observed that at all the investigated concentrations, the Pauli contribution is not significant and the EPR intensities can be well described only considering the Curie term. This result highlights two important points. First, at the studied concentrations, the ZnPc polarons do not have any metallic character and behave like isolated spin-bearing species. This behaviour is typical of moderately doped organic semiconductors..$^{25}$ Second, the absence of other contributions besides that of the Curie term underlines that the number of paramagnetic species $(N)$ does not vary with temperature in the investigated temperature range. In the presence of a different number of spin-bearing species at different temperatures, the linear trend of EPR intensity as a function of $1 / T$ would be lost. The latter appears particularly important since it suggests that the formation of the CT states is temperature independent. This result is in excellent agreement with the recent optical measurements by Tietze et al., confirming that the first step of the two-step model is temperature independent. ${ }^{6}$

\subsection{Thermal activation energies for polaron transport}

In contrast to the ground-state charge transfer (ionization) step, the separation of the CT state and the mobility of the charge carriers are dependent on temperature. To shed light into this aspect, we first investigated the EPR line widths of our samples at different temperatures. Analogously to the previous section, we focused our attention only on the ZnPc positive polaron 
contribution. The peak-to-peak line widths of the EPR spectra of $\mathrm{ZnPc}$ positive polarons are reported in Fig. 4b. At all temperatures, the EPR line widths decrease at increasing doping concentrations confirming the motional narrowing regime already observed at $280 \mathrm{~K}$. Notably, a similar trend can be observed as a function of temperature. At increasing temperature, the EPR line width decreases, suggesting an average increase in the microscopic mobility $\left(\mu_{\mathrm{EPR}}\right)$ of the positive polarons. It might be worth mentioning here that the trend in EPR line width as a function of temperature can be explained either by charge hopping (mobile spin picture) or it could equally be due to dynamic fluctuations of the environment for a single charge sitting on the same molecule (static spin picture). The Lorentzian line shape with no hyperfine splitting is supporting the former hypothesis that the ZnPc positive polarons are mobile species. Indeed, $\mathrm{ZnPc}$ molecules possess many magnetic nuclei such as hydrogen $\left(I=1 / 2\right.$ for $\left.{ }^{1} \mathrm{H}\right)$ and nitrogen $\left(I=1\right.$ for $\left.{ }^{14} \mathrm{~N}\right)$ atoms which should show strong hyperfine coupling with the polaron spin. The absence of any hyperfine broadening in the line shape as confirmed by our best fit spectral simulations is in favour of the mobile spin picture which is discussed in the following.

With the aim to get a deeper insight into the microscopic mechanism of thermally-activated hopping, we focus our attention on the $\mathrm{ZnPc}$ film doped with $3 \mathrm{wt} \%(\mathrm{MR}=0.052)$ of F6-TCNNQ. We chose the $3 \mathrm{wt} \%$ sample as a representative case as it features an intermediate doping concentration in the range investigated. In Fig. SI16 (ESI $\dagger$ ), we report the temperature-dependent EPR spectra for our reference ZnPc sample (doped with F6-TCNNQ at $3 \mathrm{wt} \%$ ). From best fit spectral simulations, we obtained the FWHM of the EPR contribution from ZnPc polarons. These are plotted on a logarithmic scale as a function of the inverse of the temperature in Fig. 4c. We decided to fit the observed trend to provide a rough estimate of the activation barrier for the microscopic transport mechanism of the charge carriers in our doped films. From the linear fit, we obtain an activation barrier of $(5 \pm 1) \mathrm{meV}$ that is similar to activation barriers probed via EPR reported for different polymers. $^{30}$ The observed low value of activation energy suggests that in our samples the polaron microscopic transport takes place through the hopping of carriers from one localized state to another, differently from a transport mechanism based on extended states with periodic trapping-detrapping in localized states. To have a closer look at this result, we carried out temperature-dependent conductivity measurements performed on the same $3 \mathrm{wt} \%(\mathrm{MR}=0.052)$ doped $\mathrm{ZnPc}$ samples, shown in Fig. 4d. The measurements can be fitted using an Arrhenius law with an activation energy of $233 \pm 1 \mathrm{meV}$. This is in close agreement with values reported in the literature and $\sim 40$ times larger than the EPR value. ${ }^{7}$ The activation energy measured from electrical measurements is the sum of the CT state binding energy and the hole transport activation energy. The CT binding is the dominant contribution to the activation energy of conductivity, as demonstrated by Schwarze et al. who indeed found a clear dependence of the thermallyactivated conductivity on CT state dissociation. ${ }^{7}$ This result has been recently further corroborated by a computational analysis indicating a Coulomb binding energy for the CT state of similar zinc-phthalocyanine systems in the range of $\sim 200-$ $300 \mathrm{meV} .{ }^{50}$ We are thus left to conclude that the hole transport contribution to the activation energy is minor. It is important to keep in mind that the activation energy for charge hopping is the sum of two terms, one that depends on the standard deviation of the (Gaussian) static disorder, $\sigma$, of the form $\sigma^{2} /$ $k T$, and one that scales with the polaron relaxation energy, as $\lambda /$ $4 .^{51}$ Since the EPR line width probes polaron transport at the microscopic level, the static disorder is likely negligible as ZnPc is polycrystalline and the order within the crystalline grains is high. This result has been also confirmed by Grazing Incidence Wide Angle X-ray Scattering (GIWAXS) measurements reported in the SI-12 (ESI $\dagger$ ). The remaining term is the classical (lowfrequency) part of $\lambda$, which in rigid molecules like ZnPc is very small. We note that an accurate assessment of the external reorganization energy in acenes has been calculated to be $\sim 40$ $\mathrm{meV},{ }^{52}$ which translates into an activation energy for polaron transport of $10 \mathrm{meV}$, in agreement with the activation energy provided by EPR measurements.

All in all, our EPR analysis sheds light into the thermallyactivated polaron transport at microscopic level from a "spincentered" perspective. Most importantly, the activation barrier extracted from the EPR measurements is very low $(\sim 5 \mathrm{meV})$ and thus confirms that the much larger energy activation associated to electrical conductivity measurements is in fact dictated by dissociation of the bound polarons. Thus, EPR demonstrates to be an handy tool in order to obtain direct information on polaron hopping transport, which is difficult to

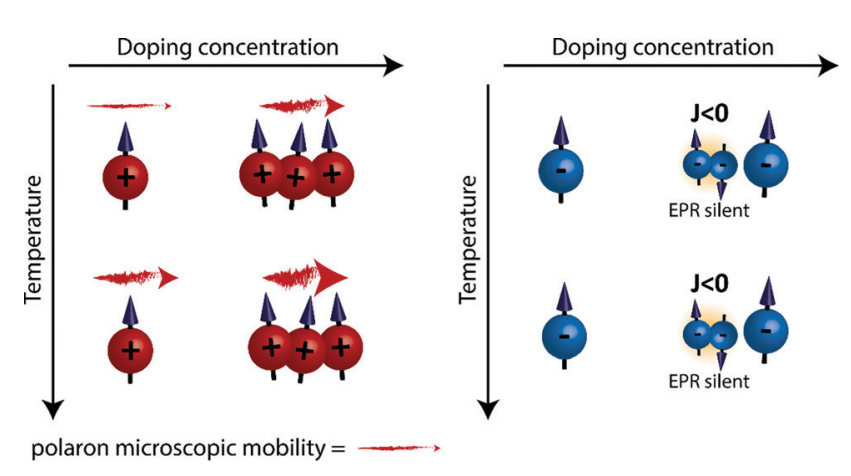

Fig. 5 Schematic representation of ZnPc p-doped with F6-TCNNQ from the electron spins' point of view. After the single electron transfer, two spin-bearing species are generated: a positive polaron localized on the $\mathrm{ZnPc}$ (red charge, figure on the left hand side) and a negative anion localized on the F6-TCNNQ (blue charge, figure on the right hand side). At higher doping concentrations, the EPR intensity (and then the number) of the polarons increases monotonically, while the EPR intensity of the anions show a maximum at $3 w t \%(M R=0.052)$ doping concentration as an effect of an antiferromagnetic coupling mechanism occurring at high doping concentrations. At different temperatures, the number of the spinbearing species does not change, underlying a temperature-independent electron transfer at the basis of charge generation in doped films. The microscopic polaron transport shows a positive trend as a function of doping concentration and temperature, which is confirmed by conductivity measurements. The red arrow thickness is proportional to the microscopic mobility. 
obtain with other techniques. Although further analysis is needed, this rationale can be extended to most of the polycrystalline organic semiconductors based on evaporated small molecules.

\section{Conclusions}

Our work sheds light into the equilibrium and the transport dynamics of the species generated in the molecular doping of organic semiconductors by taking advantage of their electron spin. The analysis was possible thanks to the use of electron paramagnetic resonance (EPR) spectroscopy, which provides the fundamental advantage of directly detecting all the spinbearing species generated in the doping process, combined with electrical measurements and theoretical DFT calculations. The investigation performed on a model system composed of a layer of ZnPc as a host, p-doped with the acceptor F6-TCNNQ, highlights the presence of two spin-bearing species $(S=1 / 2)$, whose $g$-values are attributable to the F6-TCNNQ radical anion $\left(g_{\text {iso }}=2.0034 \pm 0.0005\right)$ and the $\mathrm{ZnPc}$ positive polaron $\left(g_{\text {iso }}=\right.$ $2.0023 \pm 0.0005)$. This result confirms the presence of a full electron transfer event at the basis of the doping process. The systematic analysis carried out at different doping concentrations $(0-5 \mathrm{wt} \%, \mathrm{MR}=0-0.085)$ and temperatures $(80-280 \mathrm{~K})$ clarifies the generation of the charge carriers and the polaronic transport at microscopic level in doped organic semiconductors, as schematized in Fig. 5. At higher doping concentrations, a monotonic increase in the number of ZnPc positive polarons is derived from quantitative analysis of the EPR data. Conversely, a non-monotonic trend with a maximum at $3 \mathrm{wt} \%$-doping concentration is observed for the F6-TCNNQ anion. Based on quantum-chemical modelling, we propose that an antiferromagnetic coupling between the electron spins prevails at higher doping concentrations. This antiferromagnetic coupling mechanism occurring also at room temperature and devicerelevant doping concentrations $(\sim$ few wt $\%)$ suggests the presence of dopant clustering in doped polycrystalline films based on small organic molecules and could pave the way for new technological applications of doped layers in the burgeoning field of organic spintronics. ${ }^{53}$ The investigation of the microscopic polaron transport $\left(\mu_{\mathrm{EPR}}\right)$, as derived by EPR line width, suggests that charge carriers move via a thermally activated hopping process between localized states. In particular, a positive trend in the mobility as a function of increasing temperature confirms that hole polarons migrate through a thermally activated hopping process $\left(E_{\mathrm{A}}=5 \pm\right.$ $1 \mathrm{meV})$. Temperature-dependent conductivity measurements show a much larger thermal activation energy $\left(E_{\mathrm{A}}=233 \pm\right.$ $1 \mathrm{meV}$ ), which is dominated by CT state dissociation but also possibly includes a contribution from static energetic disorder (grain boundaries, etc.). This result is likely extensible to other polycrystalline semiconductors based on small molecules and suggest that further EPR analysis will allow to disclose general design rules for polaron transport in organic semiconductors.

In summary, the models and analysis developed in this work demonstrate that temperature-dependent EPR is a highly sensitive method that by itself can provide a wealth of information on doped organic systems. This information includes the doping efficiency, the nature of formed mobile polarons and static anions, the microscopic polaron mobility, the activation energies for microscopic charge transport, the spin coupling, and the indication for dopant clustering. The latter bears high technological significance when doped layers are applied in functional devices such as solar cells or OLEDs. Such information is relevant for fundamental research into the doping mechanism of organic semiconductors, opens new avenues in the field of organic spintronics, and for technological applications requiring excellent control over doping processes.

\section{Data availability}

All data is available on our research group's website or from the corresponding authors upon request.

\section{Author contributions}

The project was conceived and designed by AP with significant input from RW, GL, DB and MR. In addition, AP fabricated the EPR samples, performed the EPR measurements and their analysis. RW fabricated samples for electrical measurements, performed the conductivity analysis and developed the statistical model for inhomogeneous dopants distribution. GL carried out all the theoretical DFT simulations and discussion and wrote the theoretical part. PK provided fruitful discussion on PDS measurements, dopants distribution and film morphology, and electrical transport physics of organic semiconductors. JL performed the temperature dependent conductivity measurements. AEL supervised the diffraction experiments and analysed the GIWAXS images. OT performed the PDS measurements. AS contributed with helpful discussion of the EPR results. MR, DB and AA supervised the work and contributed with fruitful discussions. The first manuscript draft was prepared by AP. All the authors reviewed the manuscript and participated actively in the discussion of the results.

\section{Conflicts of interest}

The authors declare no competing financial interests.

\section{Acknowledgements}

This work was supported by European Union's Horizon 2020 Research and Innovation Programme under Marie Sklodowska Curie Grant Agreement No. 722651 (SEPOMO Project) and by the EPSRC (EP/L011972/1). We thank the Centre for Advanced Electron Spin Resonance (CAESR), Department of Chemistry, University of Oxford, for EPR measurements. AP would like to thank Dr William Myers, CAESR facility, for help with EPR measurements. RW acknowledges EPSRC for the funding through the Centre for Doctoral Training in Plastic Electronics (EP/L016702/1). PK and MR acknowledge funding from the Global Challenges Research Fund (GCRF) through Science \& 
Technology Facilities Council (STFC), grant number ST/ R002754/1: Synchrotron Techniques for African Research and Technology (START). GL is indebted to Prof. Federico Totti and Dr Matteo Briganti for useful discussions on DFT calculations. Computational resources were provided by the Consortium des Équipements de Calcul Intensif (CÉCI), funded by the Fonds de la Recherche Scientifiques de Belgique (F.R.S.-FNRS) under Grant No. 2.5020.11, as well as the Tier-1 supercomputer of the Fedération Wallonie-Bruxelles, infrastructure funded by the Walloon Region under Grant Agreement No. 1117545. DB is a FNRS Research Director. AEL thanks the EPSRC for funding through the Doctoral Training Partnership EP/N509711/1 as well as the STFC, ISIS Neutron and Muon facility and project 1948713. The GIWAXS data was collected during experiment SI20426-1 at beamline I07 of the Diamond Light Source.

\section{References}

1 B. Lüssem, M. Riede and K. Leo, Phys. Status Solidi A, 2013, 210, 9-43.

2 B. Lussem, C. M. Keum, D. Kasemann, B. Naab, Z. Bao and K. Leo, Chem. Rev., 2016, 116, 13714-13751.

3 K. Walzer, B. Maennig, M. Pfeiffer and K. Leo, Chem. Rev., 2007, 107, 1233-1271.

4 S. J. Yoo and J. J. Kim, Macromol. Rapid Commun., 2015, 36, 984-1000.

5 D. Kiefer, R. Kroon, A. I. Hofmann, H. Sun, X. Liu, A. Giovannitti, D. Stegerer, A. Cano, J. Hynynen, L. Yu, Y. Zhang, D. Nai, T. F. Harrelson, M. Sommer, A. J. Moule, M. Kemerink, S. R. Marder, I. McCulloch, M. Fahlman, S. Fabiano and C. Muller, Nat. Mater., 2019, 18, 149-155.

6 M. L. Tietze, J. Benduhn, P. Pahner, B. Nell, M. Schwarze, H. Kleemann, M. Krammer, K. Zojer, K. Vandewal and K. Leo, Nat. Commun., 2018, 9, 1182.

7 M. Schwarze, C. Gaul, R. Scholz, F. Bussolotti, A. Hofacker, K. S. Schellhammer, B. Nell, B. D. Naab, Z. Bao, D. Spoltore, K. Vandewal, J. Widmer, S. Kera, N. Ueno, F. Ortmann and K. Leo, Nat. Mater., 2019, 18, 242-248.

8 M. Schwarze, W. Tress, B. Beyer, F. Gao, R. Scholz, C. Poelking, K. Ortstein, A. A. Günther, D. Kasemann, D. Andrienko and K. Leo, Science, 2016, 352, 1446-1449.

9 I. Salzmann, G. Heimel, M. Oehzelt, S. Winkler and N. Koch, Acc. Chem. Res., 2016, 49, 370-378.

10 H. Mendez, G. Heimel, S. Winkler, J. Frisch, A. Opitz, K. Sauer, B. Wegner, M. Oehzelt, C. Rothel, S. Duhm, D. Tobbens, N. Koch and I. Salzmann, Nat. Commun., 2015, 6, 8560 .

11 I. Salzmann, G. Heimel, S. Duhm, M. Oehzelt, P. Pingel, B. M. George, A. Schnegg, K. Lips, R. P. Blum, A. Vollmer and N. Koch, Phys. Rev. Lett., 2012, 108, 035502.

12 H. Jiang, P. Hu, J. Ye, Y. Li, H. Li, X. Zhang, R. Li, H. Dong, W. Hu and C. Kloc, Adv. Mater., 2017, 29, 1605053.

13 P. Pingel, M. Arvind, L. Kölln, R. Steyrleuthner, F. Kraffert, J. Behrends, S. Janietz and D. Neher, Adv. Electron. Mater., 2016, 2, 1600204.
14 G. Zuo, X. Liu, M. Fahlman and M. Kemerink, Adv. Funct. Mater., 2018, 28, 1703280.

15 P. Li, G. Ingram, J.-J. Lee, Y. Zhao and Z.-H. Lu, Commun. Phys., 2019, 2, 2.

16 J. Li, I. Duchemin, O. M. Roscioni, P. Friederich, M. Anderson, E. Da Como, G. Kociok-Köhn, W. Wenzel, C. Zannoni, D. Beljonne, X. Blase and G. D'Avino, Mater. Horiz., 2019, 6, 107-114.

17 M. L. Tietze, P. Pahner, K. Schmidt, K. Leo and B. Lüssem, Adv. Funct. Mater., 2015, 25, 2701-2707.

18 J. Li, G. D’Avino, A. Pershin, D. Jacquemin, I. Duchemin, D. Beljonne and X. Blase, Phys. Rev. Mater., 2017, 1, 025602.

19 J. Gao, E. T. Niles and J. K. Grey, J. Phys. Chem. Lett., 2013, 4, 2953-2957.

20 J. Gao, B. W. Stein, A. K. Thomas, J. A. Garcia, J. Yang, M. L. Kirk and J. K. Grey, J. Phys. Chem. C, 2015, 119, 16396-16402.

21 R. Fujimoto, Y. Yamashita, S. Kumagai, J. Tsurumi, A. Hinderhofer, K. Broch, F. Schreiber, S. Watanabe and J. Takeya, J. Mater. Chem. C, 2017, 5, 12023-12030.

22 T. Schneider, F. Limberg, K. Yao, A. Armin, N. Jürgensen, J. Czolk, B. Ebenhoch, P. Friederich, W. Wenzel, J. Behrends, H. Krüger and A. Colsmann, J. Mater. Chem. C, 2017, 5, 770-776.

23 R. Steyrleuthner, Y. Zhang, L. Zhang, F. Kraffert, B. P. Cherniawski, R. Bittl, A. L. Briseno, J. L. Bredas and J. Behrends, Phys. Chem. Chem. Phys., 2017, 19, 3627-3639.

24 A. Aguirre, P. Gast, S. Orlinskii, I. Akimoto, E. J. J. Groenen, H. El Mkami, E. Goovaerts and S. Van Doorslaer, Phys. Chem. Chem. Phys., 2008, 10, 7129-7138.

25 K. Kang, S. Watanabe, K. Broch, A. Sepe, A. Brown, I. Nasrallah, M. Nikolka, Z. Fei, M. Heeney, D. Matsumoto, K. Marumoto, H. Tanaka, S. Kuroda and H. Sirringhaus, Nat. Mater., 2016, 15, 896-902.

26 H. Tanaka, M. Hirate, S. Watanabe and S. Kuroda, Adv. Mater., 2014, 26, 2376-2383.

27 H. Matsui, T. Hasegawa, Y. Tokura, M. Hiraoka and T. Yamada, Phys. Rev. Lett., 2008, 100, 126601.

28 K. Marumoto, N. Arai, H. Goto, M. Kijima, K. Murakami, Y. Tominari, J. Takeya, Y. Shimoi, H. Tanaka, S.-I. Kuroda, T. Kaji, T. Nishikawa, T. Takenobu and Y. Iwasa, Phys. Rev. B: Condens. Matter Mater. Phys., 2011, 83, 075302.

29 A. Gasonoo, J. M. Kim, S. J. Yoo, Y. J. Cho, J. H. Lee, Y. Choi, J. J. Kim and J. H. Lee, Org. Electron., 2019, 67, 242-246.

30 H. Matsui, D. Kumaki, E. Takahashi, K. Takimiya, S. Tokito and T. Hasegawa, Phys. Rev. B: Condens. Matter Mater. Phys., 2012, 85, 035308.

31 J. Niklas and O. G. Poluektov, Adv. Energy Mater., 2017, 7, 1602226.

32 S. Stoll and A. Schweiger, J. Magn. Reson., 2006, 178, 42-55. 33 C. Adamo and V. Barone, J. Chem. Phys., 1999, 110, 6158-6170.

34 F. Neese, Wiley Interdiscip. Rev.: Comput. Mol. Sci., 2012, 2, 73-78.

35 A. Bencini and F. Totti, J. Chem. Theory Comput., 2009, 5, 144-154. 
36 C. J. Boyle, M. Upadhyaya, P. Wang, L. A. Renna, M. Lu-Díaz, S. Pyo Jeong, N. Hight-Huf, L. Korugic-Karasz, M. D. Barnes, Z. Aksamija and D. Venkataraman, Nat. Commun., 2019, 10, 2827.

37 W. B. Jackson, N. M. Amer, A. C. Boccara and D. Fournier, Appl. Opt., 1981, 20, 1333-1344.

38 C. Nicklin, J. Martinez-Hardigree, A. Warne, S. Green, M. Burt, J. Naylor, A. Dorman, D. Wicks, S. Din and M. Riede, Rev. Sci. Instrum., 2017, 88, 103901.

39 R. Warren, A. Privitera, P. Kaienburg, A. E. Lauritzen, O. Thimm, J. Nelson and M. K. Riede, Nat. Commun., 2019, 10, 5538.

40 S. Schaefer, PhD thesis, Freie Universität Berlin, 2010.

41 A. L. Sutton, B. F. Abrahams, D. M. D'Alessandro, T. A. Hudson, R. Robson and P. M. Usov, CrystEngComm, 2016, 18, 8906-8914.

42 Y. Karpov, T. Erdmann, M. Stamm, U. Lappan, O. Guskova, M. Malanin, I. Raguzin, T. Beryozkina, V. Bakulev, F. Günther, S. Gemming, G. Seifert, M. Hambsch, S. Mannsfeld, B. Voit and A. Kiriy, Macromolecules, 2017, 50, 914-926.

43 J. A. Weil, J. R. Bolton and J. E. Wertz, Electron Paramagnetic Resonance: Elementary Theory and Practical Applications, Wiley, 1994.

44 V. I. Krinichnyi, Multi frequency EPR spectroscopy of conjugated polymers and their nanocomposites, CRC Press, 2017.
45 R. Kubo and K. Tomita, J. Phys. Soc. Jpn., 1954, 9, 888-919. 46 L. Goris, K. Haenen, M. Nesládek, P. Wagner, D. Vanderzande, L. De Schepper, J. D'haen, L. Lutsen and J. V. Manca, J. Mater. Sci., 2005, 40, 1413-1418.

47 S.-W. Feng, M.-C. Shih, C. J. Huang and C.-T. Chung, Thin Solid Films, 2009, 517, 2719-2723.

48 L. Noodleman, J. Chem. Phys., 1981, 74, 5737-5743.

49 J. S. Miller, Acc. Chem. Res., 2007, 40, 189-196.

50 A. Privitera, G. Londi, M. Riede, G. D'Avino and D. Beljonne, Adv. Funct. Mater., 2020, 30, 2004600.

51 H. Bässler, P. M. Borsenberger and R. J. Perry, J. Polym. Sci., Part B: Polym. Phys., 1994, 32, 1677-1685.

52 N. G. Martinelli, J. Idé, R. S. Sánchez-Carrera, V. Coropceanu, J.-L. Brédas, L. Ducasse, F. Castet, J. Cornil and D. Beljonne, J. Phys. Chem. C, 2010, 114, 20678-20685.

53 S.-J. Wang, D. Venkateshvaran, M. R. Mahani, U. Chopra, E. R. McNellis, R. Di Pietro, S. Schott, A. Wittmann, G. Schweicher, M. Cubukcu, K. Kang, R. Carey, T. J. Wagner, J. N. M. Siebrecht, D. P. G. H. Wong, I. E. Jacobs, R. O. Aboljadayel, A. Ionescu, S. A. Egorov, S. Mueller, O. Zadvorna, P. Skalski, C. Jellett, M. Little, A. Marks, I. McCulloch, J. Wunderlich, J. Sinova and H. Sirringhaus, Nat. Electron., 2019, 2, 98-107. 